\title{
COVID-19 and its sequelae: a platform for optimal patient care, discovery and training
}

\author{
Richard C. Becker ${ }^{1}$ \\ Accepted: 5 January 2021 / Published online: 27 January 2021 \\ (c) The Author(s), under exclusive licence to Springer Science+Business Media, LLC part of Springer Nature 2021
}

\begin{abstract}
COVID-19- related patient care and research have focused on short-term outcomes, particularly among those with underlying or preexisting medical conditions. A major focus has been on mortality rates. Broadening the dialogue is neither meant nor intended to disparage the near-term devastation felt globally each day, but rather to begin preparation for optimally caring for and addressing the needs of survivors. The sequelae of COVID-19 includes acute, subacute and chronic stages of the condition. If one applies current World Health Organization (WHO) statistics to calculate the global burden of disease, there are 98,000,000 COVID-19 survivors. The following editorial focuses on post-COVID sequelae as a continuum of patient care needs, as well as discovery and training opportunities in an academic setting.
\end{abstract}

Keywords COVID-19 sequelae $\cdot$ Long-COVID-19 syndrome

\section{Highlights}

- COVID-19 has multiple stages and phases.

- The sequelae of COVID-19 can affect any organ system.

- Efforts and investigation in the medical and scientific communities must focus on predisposing factors, phenotypes, diagnosis menus, prevention and treatment.

\section{Introduction}

At the time of this writing, 21 January 2021, there have been over 100 million cases and 2 million deaths reported since the start of the 2019 Coronavirus (CoV) (SARS-severe acute respiratory syndrome $\mathrm{CoV}-2$ ) (SARS-CoV-2) pandemic (www.who.int/covid; accessed 21 January 2021). The number of new cases has been increasing at a rate of 5 million per week. In the past week, the two countries reporting the highest number of cases were the United States of America (reporting over 1.5 million cases, a 11\% increase from the

Richard C. Becker

Richard.becker@uc.edu

1 Heart, Lung and Vascular Institute, University of Cincinnati College of Medicine, 231 Albert Sabin Way, Cincinnati, OH 45267, USA previous week) and Brazil (over 379,000 new cases, a 21\% increase) (Fig. 1) (Table 1).

COVID-19 patient care and research have focused on short-term outcomes, particularly among those with underlying or preexisting medical conditions. A major focus has been on mortality rates. This is understandable and fitting as the impact, at a personal and societal level is without recent precedent. Broadening the dialogue is neither meant nor intended to disparage the near-term devastation felt globally each day, but rather to begin preparation for optimally caring for and addressing the needs of survivors (reviewed in Becker) [1, 2]. The following editorial focuses on postCOVID sequelae as a continuum of patient care needs, as well as discovery and training opportunities in an academic setting.

\section{COVID-19 sequelae}

The sequelae of COVID-19 includes acute, subacute and chronic stages of the condition. Up to $20 \%$ of patients surviving an initial hospitalization require readmission within 60 days of discharge [3]. The most common readmission diagnoses are COVID-19 (30.2\%), sepsis (8.5\%), pneumonia $(3.1 \%)$ and heart failure (3.1\%). Nearly one-in-four patients require ICU-level care and the overall mortality rate approaches $20.0 \%$. 


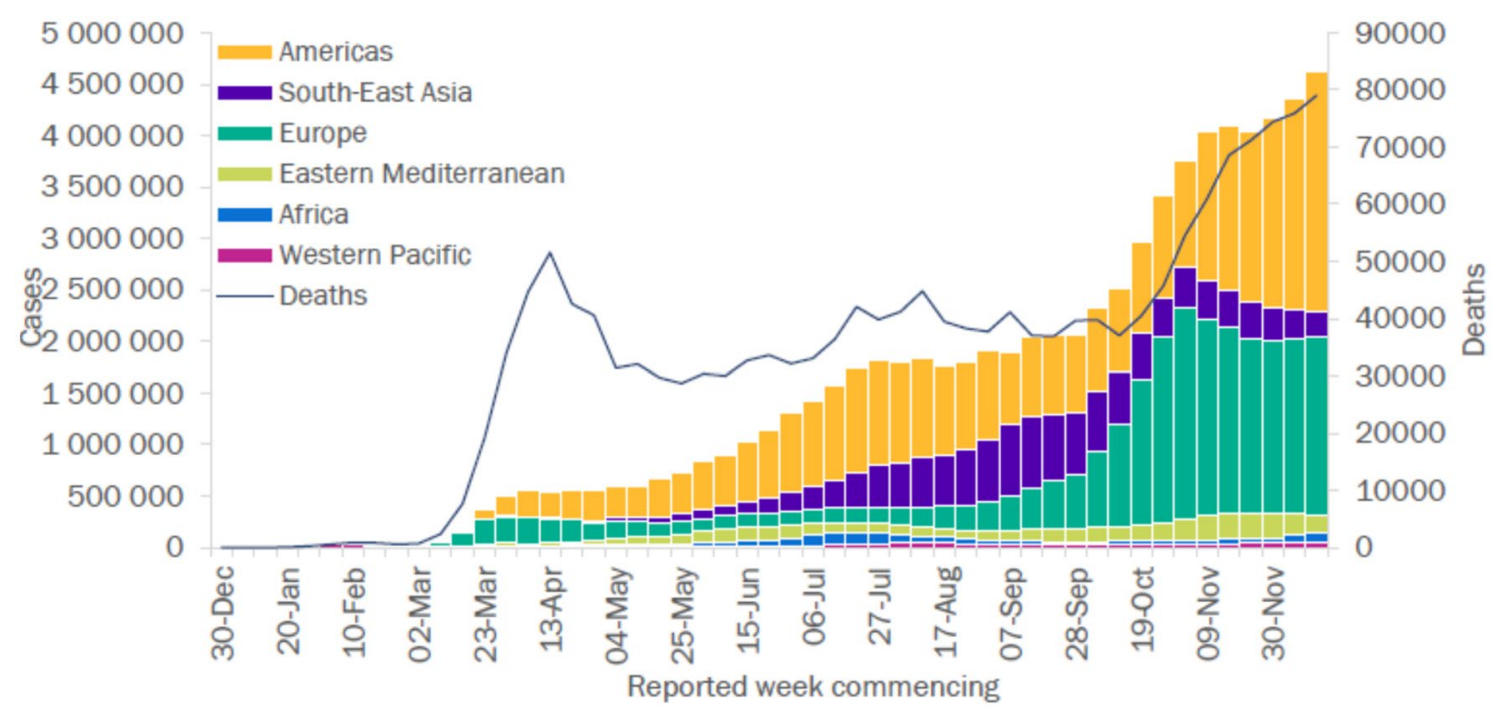

Fig. 1 COVID-19 cases reported weekly by World Health Organization region as of 20 December 2020. www.who.int/covid; accessed 20 December 2020

Table 1 Newly reported and cumulative COVID-19 confirmed cases and deaths according to World Health Organization region as of 20 December 2020

\begin{tabular}{|c|c|c|c|c|c|c|}
\hline WHO region & $\begin{array}{l}\text { New cases in last } \\
7 \text { days }(\%)\end{array}$ & $\begin{array}{l}\text { Change in new } \\
\text { cases in last } \\
7 \text { days* }\end{array}$ & $\begin{array}{l}\text { Cumulative cases } \\
(\%)\end{array}$ & $\begin{array}{l}\text { New deaths in } \\
\text { last } 7 \text { days }(\%)\end{array}$ & $\begin{array}{l}\text { Change in new } \\
\text { deaths in last } \\
7 \text { days* }\end{array}$ & $\begin{array}{l}\text { Cumulative deaths } \\
(\%)\end{array}$ \\
\hline Americas & $2,321,202(50 \%)$ & $13 \%$ & $32,437,597(43 \%)$ & $32,397(41 \%)$ & $9 \%$ & $809,105(48 \%)$ \\
\hline Europe & $1,726,941(37 \%)$ & $2 \%$ & $23,691,857(32 \%)$ & $36,286(46 \%)$ & $3 \%$ & $522,719(31 \%)$ \\
\hline South-East Asia & $249,007(5 \%)$ & $-14 \%$ & $11,610,444(15 \%)$ & $3968(5 \%)$ & $-10 \%$ & $176,826(11 \%)$ \\
\hline $\begin{array}{l}\text { Eastern Mediter- } \\
\text { ranean }\end{array}$ & $174,325(4 \%)$ & $-14 \%$ & $4,665,285(6 \%)$ & $3852(5 \%)$ & $-12 \%$ & $115,495(7 \%)$ \\
\hline Africa & $94,653(2 \%)$ & $27 \%$ & $1,716,697(2 \%)$ & $1862(2 \%)$ & $34 \%$ & $37,741(2 \%)$ \\
\hline Western Pacific & $\begin{array}{l}46,662 \\
(1 \%)\end{array}$ & $3 \%$ & $1,006,682(1 \%)$ & $636(1 \%)$ & $18 \%$ & $18,895(1 \%)$ \\
\hline Global & $4,612,790(100 \%)$ & $6 \%$ & $75,129,306(100 \%)$ & $79,001(100 \%)$ & $4 \%$ & $1,680,794(100 \%)$ \\
\hline
\end{tabular}

www.who.int/covid; accessed 20 December 2020

*Compared with the previous 7 days

Emerging data show that up to $70 \%$ of low-risk patients (40-50 years of age without pre-existing hypertension, diabetes mellitus, obesity or heart disease) with COVID19 have symptoms, including fatigue, impaired stamina, breathlessness, headaches and muscle aches 4 - to- 5 months following the initial infection. Total body quantitative magnetic resonance imaging reveals organ impairment involving the heart (32\%), lungs (33\%), kidneys (12\%), liver (10\%), pancreas (17\%), and spleen (6\%). Multi-organ impairment occurs in $25 \%$ of patients [4].

If one applies current World Health Organization (WHO) statistics to calculate the global burden of disease, there are 73,400,000 COVID-19 survivors (www.who.int/covid; accessed 20 December 2020). An ability to advance the field's understanding requires answers to several fundamental questions. What are the stages, phases and variants of COVID-19? Is there a standardized nomenclature? What represents an optimal diagnostic menu for evaluating survivors? What is the ideal means and duration for follow-up? The overarching objectives of the following editorial are to (1) discuss the cause and natural history of Long COVID19 syndrome (2) consider an efficient and cost-effective diagnostic approach for evaluation, and (3) posit discovery platforms, optimal treatment and longitudinal followup strategies and training opportunities for clinicians and physician-scientists. 


\section{Proposed definitions and criteria for COVID-19}

The University of Cincinnati Heart, Lung and Vascular Institute COVID-19 clinic began evaluating patients in June of 2020. We have established diagnostic criteria, testing menus, hospital-based processes of care and follow-up strategies. Similar to other groups, we believe that frameworks and timelines of illness are of critical value to clinicians, researchers and the public [5].

Patients with moderate-to-high acuity COVID-19 requiring hospitalization and advanced care undergo evaluation in the Post-ICU Clinic by a team of cardiologists, pulmonologists, critical care specialists, physical therapists, occupational therapists, speech therapists and a pharmacist. In a majority of cases, initial care took place in either the Medical or Cardiovascular Intensive Care Unit (ICU) or Medical Step-Down Unit. Patients have their initial visit within 2 weeks of hospital discharge. Many of our high-acuity patients will already be undergoing rehabilitation, with physical, occupational and speech therapy in a dedicated long-term acute care facility.

Patients with low-acuity COVID-19, including those without a medical indication for hospitalization or who experienced minimal symptoms at the time of initial infection, but are experiencing persistent symptoms are evaluated in the COVID-19 Clinic (Table 2).

We support the development of definitions and criteria that exist under an umbrella of COVID-19 sequelae, and acknowledge an equally broad implication of terminology such as Long-COVID-19 syndrome. We also favor a specific classification that facilitates updates as new knowledge comes forward for several reasons: first, the evidence to date supports distinct phenotypes among survivors. Second, establishing registries, research platforms and procuring funds for research endeavors are more likely when phenotypes are well defined. Third, providing and documenting optimal management are difficult when patients exist in large, heterogeneous groups. Fourth, education of the lay and medical communities depends on clear and consistent terms.

The multi-disciplinary COVID-19 Clinic located at The University of Cincinnati Medical Center has adopted a dichotomous initial classification to facilitate timely and focused patient care (Fig. 2, Table 3).

Table 2 General acuity criteria for patients with COVID-19

\section{Mild illness}

Individuals who have any of various signs and symptoms of COVID-19 (e.g., fever, cough, sore throat, malaise, headache, muscle pain) without shortness of breath, dyspnea, or abnormal chest imaging

Moderate illness

Individuals who have evidence of lower respiratory tract disease by clinical assessment or imaging, and a saturation of oxygen $\left(\mathrm{SpO}_{2}\right) \geq 94 \%$ on room air

Severe illness

Individuals who have respiratory frequency $>30$ breaths per minute, $\mathrm{SpO}_{2}<94 \%$ on room air (or, for patients with chronic hypoxemia, a decrease from baseline of $>3 \%$ ), ratio of arterial partial pressure of oxygen to fraction of inspired oxygen $\left(\mathrm{PaO}_{2} / \mathrm{FiO}\right)_{2}<300 \mathrm{mmHg}$, or lung infiltrates $>50 \%$

Critical illness

Individuals who have respiratory failure, septic shock, cardiogenic shock and/or multiple organ dysfunction

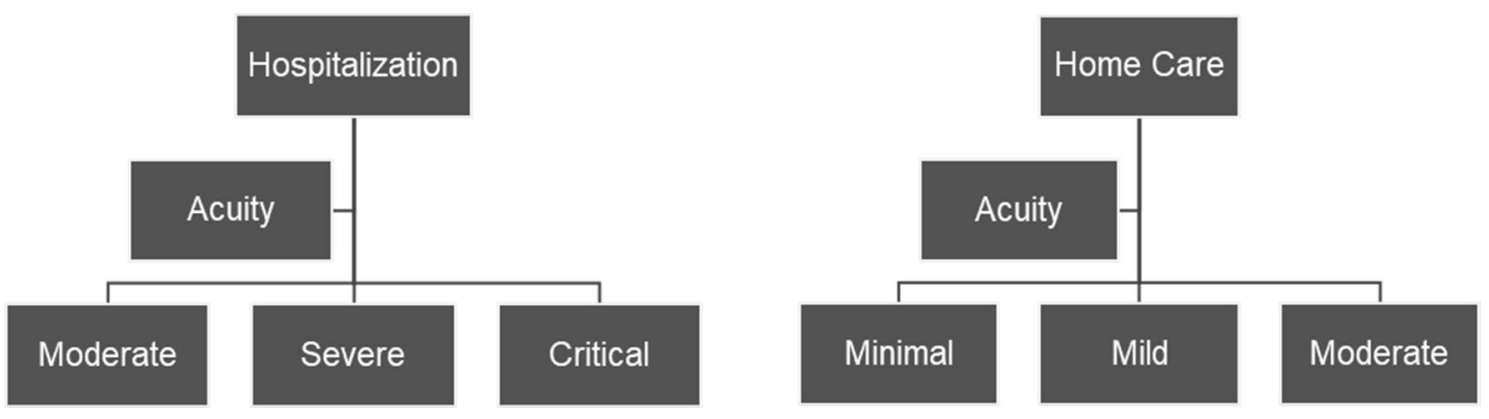

Fig. 2 Clinical criteria for patients with COVID-19 according to site of care (hospital or home) and acuity (see text for follow-up care algorithm) 
Table 3 Proposed COVID-19 sequelae subtype criteria

\begin{tabular}{|c|c|c|c|c|c|c|c|}
\hline & Type 1 & Type 2 & Type 3 & & Type 4 & & Type 5 \\
\hline \multirow[t]{2}{*}{ Initial symptoms } & \multirow[t]{2}{*}{ Variable $^{\mathrm{a}}$} & \multirow[t]{2}{*}{ Mild } & $\mathrm{A}$ & B & A & B & \multirow[t]{2}{*}{ None } \\
\hline & & & Mild & Mild & None & None & \\
\hline Duration of symptoms & Variable $^{\mathrm{a}}$ & $>6$ weeks & $3-6$ months & $>6$ months & Variable & Variable & N/A \\
\hline Period of quiescence & No & No & Yes & Yes & No & No & N/A \\
\hline \multirow[t]{2}{*}{ Delayed onset of symptoms } & \multirow[t]{2}{*}{ No } & \multirow[t]{2}{*}{ No } & \multirow{2}{*}{\multicolumn{2}{|c|}{ No }} & Yes & Yes & \multirow[t]{2}{*}{ Yes } \\
\hline & & & & & $\geq 3$ months & $\geq 6$ months & \\
\hline
\end{tabular}

${ }^{\mathrm{a}}$ Correlate with the severity of initial infection, number of organ system injured and pre-existing medical conditions

\section{COVID-19 sequelae subtypes}

Type 1 Type 1 Long COVID-19 syndrome includes patients with varying lengths of recovery and rehabilitation that directly correlate with the severity of infection, target organ damage and pre-existing medical conditions at the time of initial infection.

Type 2 Type 2 Long COVID-19 syndrome is characterized by symptoms persisting 6 weeks from the time of initial infection.

Type 3 Type 3 Long COVID-19 syndrome features a period of quiescence or near-complete recovery following initial infection, followed by a return of symptoms that persist for $\geq 3$ months (Type 3A) or $\geq 6$ months (Type 3B).

Type 4 Type 4 Long COVID-19 syndrome includes patients who are initially asymptomatic at the time of a positive SARS-CoV-2 test but develop symptoms beginning 1-3 months (Type 4A) or $\geq 3$ months (Type 4B) later that persist for varying lengths of time.

Type 5 Type 5 Long COVID-19 syndrome includes patients who are initially asymptomatic or minimally symptomatic at the time of a positive SARS-CoV-2 test and experience sudden death within the next 12 months. Subclinical myocarditis with fibrosis and malignant arrhythmias are likely responsible.

We expect the COVID Sequelae Subtype criteria to evolve further and include organ specific subtypes-heart and circulatory system, pulmonary system, central and peripheral nervous system, musculoskeletal system, gastrointestinal and hepatobiliary system and integumentary system (Fig. 3).

\section{Processes of care}

It is in our opinion that optimal care for persons with COVID-19 includes a follow-up evaluation. This applies to all laboratory documented cases, as well as those with compelling signs and symptoms of SARS-CoV-2 infection in the absence of testing or in some instances a negative antigenbased test or inappropriately timed (from symptom onset), polymerase chain reaction (PCR) test. Identification of

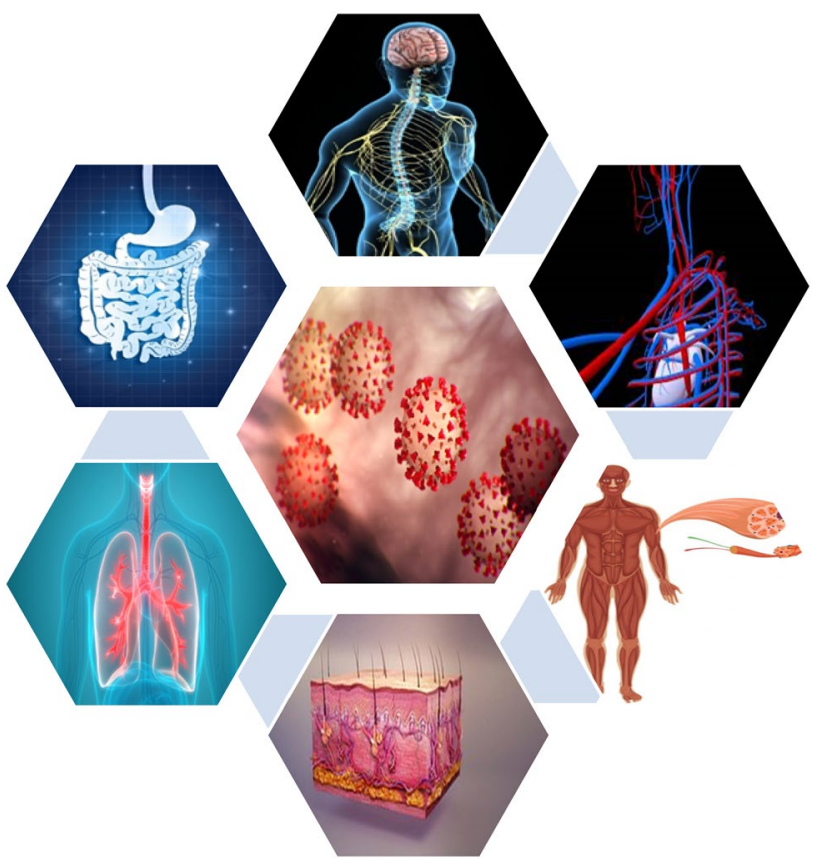

Fig. 3 The COVID-19 sequelae criteria and subtypes characterized by organ system involvement. While many survivors have symptoms originating from more than one system, others have one dominant system that is responsible for a majority of the signs, symptoms and overall phenotype. The SARS-CoV-2 virus, shown in the center is a starting point for COVID-19, but emerging data support humoral immunity, autoimmunity, poorly regulated inflammatory responses, vascular injury and dysfunction, preexisting medical conditions, genetic factors and the residual effects of acute target organ injury as determinants of protracted illness. Organs systems shown (top panel, clockwise): central and peripheral nervous system, heart and circulatory system, musculoskeletal system, integumentary system, pulmonary system and gastrointestinal-hepatobiliary system

patients prior to hospital discharge using an electronic trigger system with bedside scheduling is our favored approach. Among persons not requiring hospitalization, provider consultation and patient self-referral are coordinated through our Communications Center. 


\section{Patient assessment}

The COVID-19 program prioritizes, triages and evaluates patients according to condition acuity, site of management (hospitalized, non-hospitalized), major organ involvement, target organ injury, dominant phenotype(s), progression of disease, preexisting medical conditions, rate of recovery and access to healthcare. A menu of blood tests have been published previously [1] and includes discovery blood sampling (see below).

\section{Non-hospitalized patients}

We recommend persons with COVID-19 and mild symptoms that resolve within 5 days see their primary care provider within 2 weeks of symptom onset (www.cdc.gov). A physical examination, electrocardiogram and other diagnostic tests when indicated and discussion about direct contacts, return to work and return to physical activities are undertaken [6-8].

We instruct persons with moderate acuity symptoms managed at home to work closely with their primary care providers employing a telehealth platform. A face-to-face follow-up visit within 3 weeks of symptom onset is encouraged (www.cdc.gov).

Persons with COVID-19 managed at home with persisting symptoms beyond 6 weeks, regardless of acuity, undergo an initial screening COVID-19 clinic tele-health visit with a cardiologist and a pulmonologist. Based on the initial evaluation, a face-to-face clinic visit is considered, and a patientspecific menu of diagnostic tests planned to coincide with an onsite assessment.

\section{Hospitalized patients}

A member of the COVID-19 Management Team undertakes an evaluation of patients with COVID-19 requiring hospitalization. Each patient undergoes stratification according to disease acuity as moderate, severe or critical. All patients surviving their initial hospitalization are candidates for follow-up in the COVID-19 clinic. Those with severe or critical COVID-19 are scheduled for evaluation in the Post-ICU High Acuity Clinic.

\section{Biorepository and discovery program}

The stimulus for our COVID-19 Clinic is to address an unmet patient need and poorly understood medical condition. The Health System wide initiative is best described as a patient care discovery platform. Specifically, we aspire to provide optimal patient care by carefully evaluating patients, characterizing their signs, symptoms and clinical progression, integrating unbiased and hypothesis-based research studies and aid in the quest to answer questions about the pathophysiology of disease and its management.

\section{Operational foundation}

Biomarker-based research brings together multi-disciplinary teams to investigate the biological underpinnings of disease, disease progression, treatment response and outcomes. Most academic Institutions have a history of collecting samples and, to a lesser degree, clinical data associated with them; however, most lack a systematic approach to cataloging these assets, maintaining the collections, or managing integration with clinical information.

A potential obstacle to effectively exploiting existing resources is the common practice of having multiple rather than a common operational infrastructure. In many cases, bio-specimen collection and testing is investigator-initiated, and only selected investigators may have access to the collections. The result is numerous independent legacy biorepositories with samples. Lastly, investigators may not have the required details (i.e. IRB specified guidance) of bio-specimens collected for future research. Our COVID-19 Biorepository includes both an in-hospital and ambulatory setting component, as well as standard operating procedures and a charter for governance.

The Division of Biomedical Informatics provides a variety of computational resources, services and support to investigators. Available computational resources include the $\mathrm{i} 2 \mathrm{~b} 2$ research data warehouse for cohort identification, a cluster with more than 300 processing cores for executing computationally intensive jobs, network filers and relational database systems for secure and regulatory compliant data storage, and an array of licensed and open-source scientific software applications including statistical analysis packages.

Advances in artificial intelligence (AI) is transforming both the art and science of medicine. The challenge of extracting meaningful information from large data sets to improve patient care has stimulated exciting new science and technology to realize the value of efficient and inexpensive monitoring; to identify common and uncommon patterns of disease; and to improve healthcare quality, safety and efficiency. By systematically analyzing large data and mimicking neural learning patterns to execute automated tasks, AI technologies facilitate the early recognition of anomalous trends and patterns and support complex management decisions. The development of new AI-based methods for unraveling unique mechanistic insights at the molecular, cellular and tissue level that otherwise are not available using traditional approaches is vital for discovery. 
COVID-19 sequelae present a unique condition wherein AI and machine-based learning identifies patterns of disease as well as similarities and differences with known infectious diseases and conditions with long-term symptoms. An example is chronic fatigue syndrome, currently known as myalgic encephalomyelitis. An understanding of COVID-19 might provide insights into this condition and the reverse may be true as well $[9,10]$

\section{Training}

COVID-19 and its sequelae provide an unparalleled opportunity for training. Physical diagnosis skills, imaging interpretation, molecular medicine, clinical trial conduct and precision medicine, using a rare disease or manifestation of a new disease as a model for translational research, and multidisciplinary care coordination are a few examples. The clinical scenario offers both challenges and broad prospects along the spectrum of translation, from bench studies to the design and implementation of clinical trials, as well as the reverse spectrum of identifying rare disease phenomena and taking those discoveries back to the bench. The optimal management of long-term sequelae requires an in-depth understanding of COVID-19, its phenotypes and natural history, followed by targeted investigation and evidence-the foundation of patient care.

\section{Strategies to track COVID-19 health status}

An ability to offer optimal care for persons with COVID19 requires a strategy to track health status. A dedicated database to identify and track all documented cases within a hospital, health system, community, city or region could only be a successful undertaking if sufficient resources were available.

Proponents of large-scale self-reported information believe that such an approach to information gathering fulfills a near-term need for data. In addition, self-reported information as a tool for tracking infectious disease has precedent with influenza $[11,12]$ in the "flu near you initiative". The construct, as employed in the Coronavirus Census Collective relies upon dedicated smart phone applications and online surveys to gather information in either in a static or a longitudinal form for use as a public health, population science, data sharing or clinical research tool [11]. One of many potential deliverables is an ability to inform the public and assist the medical community simultaneously.

Self-reported data platforms can capture information on Long-COVID syndrome. Geospatial location, clustering, age, sex, ethnicity, pre-existing conditions, treatments and outcomes are a fraction of the readily available information. Several examples of self-reported data, including social media platforms include the Body Politic COVID-19 Group and the Patient-led Research for COVID-19 Group. Efforts to de-identify collected data, protect privacy and confidentiality and maintain security across participants is critical to success. In addition, access to data for analysis requires established policies, monitoring and coordination by an experienced oversite committee, preferably under the auspices of a consortium that includes infectious disease, epidemiology, data science, bioinformatics, statistical and information technology expertise.

\section{Federal and international recognition of long-COVID syndrome}

A concerted effort to understand, study and influence the incidence, duration and both personal and societal impact of COVID-19 Sequelae began with an acknowledgement that it exists. Francis Collins, MD, PhD, the 16th Director of the United States National Institutes of Health (NIH) posted information about Long-COVID-19 syndrome or Long-Haul COVID-19 on his blog (3 September 2020). The United States Centers for Disease Control and Prevention summarized the long-term effects of COVID-19 on their website (CDC.gov: accessed December 20 2020). The NIH is funding a study, "Collaborative Cohort of Cohorts for COVID-19 Research (C4R)". Participants in C4R originated from 14 long-term cohort studies conducted at 37 academic medical centers across the United States. C4R is part of the National Heart, Lung and Blood Institute (NHLBI) Collaborative Network of Networks for COVID-19 and Therapeutic Strategies (CONNECTS). The NHLBI, the National Institutes of Neurological Disorders and Stroke (NINDS), and the National Institute on Aging fund C4R.

The WHO summarized the long-term effects of COVID19 during a situation report presented on 06 September 2020. Specifically, they commented that, "symptoms may linger for weeks to months even in people with mild disease" (WHO.gov; accessed December 13, 2020). During the same briefing, the long-term health effects of Coronavirus received attention. In particular, symptoms of chronic fatigue among $40 \%$ of people recovering from SARS that persisted for several years.

\section{Status and future directions}

SARS-CoV-2 is an RNA virus responsible for the COVID19 pandemic. There are distinct stages of the infection that include acute, subacute and chronic phases. The sequelae represent a combination of the initial viral infection, humoral and inflammatory responses, target organ injury 
Table 4 Diagnostic menu, devices and rationale for use, management tools and platforms available in the Heart, Lung and Vascular Institute COVID-19 Clinic

In-touch mini telemedicine cart Telemedicine provides timely, safe, and less expensive care, where the patient does not need to be in the same room with a health care provider(s). In addition to providing routine health care services, telemedicine has consistently shown to be effective for increasing access to care in extreme conditions, such as health care crises, remote areas, or in limited resource regions

Pulse wave velocity Pulse wave velocity is a measure of arterial stiffness that predicts future cardiovascular events. COVID-19 causes blood vessel inflammation and injury. The calculation employs a non-invasive instrument, SphygmoCor CPV® (AtCor Medical, Sydney, Australia). The same instrument calculates augmentation index - a measure of arterial elasticity, function and strain on the heart in the form of afterload

Peripheral arterial tomography Peripheral artery tomography (PAT) is a measure of vascular reactivity to intrinsic and extrinsic stimulation, including blood pressure control and response to postural changes. Patients with COVID-19 have impaired vascular reactivity that predisposes to rapid changes in blood pressure associated with dizziness, presyncope or syncope. PAT is a non-invasive test performed using a tabletop instrument (EndoPat ${ }^{\circ} 2000$; Itamar Medical)

Autonomic assessment tool: pulse wave analysis The autonomic nervous system is responsible for rapid changes in heart rate, blood pressure and blood flow to vital organs. Endothelial cell inflammation and injury occur in COVID-19. The resulting autonomic dysfunction contributes to impaired stamina and exercise intolerance. Max pulse ${ }^{\circledR}$ (Long-Life Cardio; Austin, TX) is a non-invasive instrument that rapidly and reliably assess autonomic balance

Pulmonary function testing Coronavirus-2 enters the body through the nose and mouth, quickly traveling to the upper and lower portions of the lungs. A cough, shortness of breath and wheezing are common symptoms of COVID-19 and often persist for weeks to months following the initial infection. Pulmonary function tests are an invaluable assessment tool. The Pulm-One ${ }^{\circledR}$ instrument (MiniBox+) (PulmOne $\AA$, New York, NY) is the first desktop, gasless and cabinless devise that optimizes safety and complies with all COVID-19 requirements

Home spirometers Pulmonary function impairment is the most common long-term impairment following acute infection and hospitalization with COVID-19. The natural history of disease progression and the trajectory of recovery in this population requires further study. The current standard of care for these patients for longitudinal monitoring would be office-based pulmonary function testing performed approximately every 3 months. This frequency may not be adequate and more frequent office-based testing is not practical. The ability to provide patients with a portable spirometer provides a means to measure their lung function on a regular basis at home, facilitating a comprehensive and longitudinal assessment. The Zephyrx system ${ }^{\circledR}$ provides both patients and their providers with an easy-to-understand home spirometer along with access to an online portal for protected information

Pulse oximeters Similar to home spirometers, the availability of pulse oximeters for individual patients enables them to monitor their oxygenation status in a non-invasive fashion and assists providers with making decisions for supplemental oxygen and escalation of care. The Onyx ${ }^{\circledR}$ II 9550 fingertip pulse oximeter is a small, lightweight, portable device indicated for use in measuring and displaying functional oxygen saturation of arterial hemoglobin $\left(\% \mathrm{SpO}_{2}\right)$ and pulse rate of patients

Echocardiography Twenty percent of patients with COVID-19 have injury to the heart during the acute phase of illness. The available evidence shows that $>50 \%$ of patients with Long-COVID-19 syndrome have evidence of heart inflammation, placing them at risk for heart failure and serious irregularities of the heart's rhythm. Echocardiography represents the standard of care for assessment, risk stratification, medication decisions and determining a need for additional testing, such as cardiac MRI or further evaluation for pulmonary hypertension

Vital signs monitor Baseline and point-of-care monitoring of a patient's vital signs allows providers to quickly pinpoint subtle signs of patient deterioration, and proactively manage an individuals' care. Automated patient monitoring systems are an invaluable assessment tool relied upon by all providers. The Philips Healthcare EarlyVue VS30® complies with all COVID-19 requirements while providing a tool to monitor vital signs

Wrist oximeters One of the most common symptoms of Long-COVID-19 syndrome is lingering fatigue. A significant proportion of patients with preexisting medical illnesses are at increased risk of obstructive sleep apnea. The Nonan wrist oximeter ${ }^{\mathrm{TM}}$ enable continuous measurement of peripheral oxygen saturation at night, offers digital download of the data to determine the time spent in a hypoxic state while sleeping, and guides the initial evaluation and management of sleep apnea

Home sleep apnea testing devices In patients at increased risk of obstructive sleep apnea, the ability to perform sleep testing at home provides physiological data that is more reflective of real life sleep patterns. The Nox T3 System ${ }^{\circledR}$ uses Bluetooth ${ }^{\circledR}$ technology to create a wireless body area network to maximize patient comfort and has a built-in microphone, capable of recording high quality sound

Percutaneous transdermal capnograph This device is available for spot checks in the clinic, and overnight use at home or in the sleep laboratory to assess adequacy of ventilation and the need for respiratory support in patients recovering from COVID-19

and either unknown or poorly defined factors that cause prolonged symptoms stemming from damage, dysfunction or impaired regulation of one or more organ systems. The true prevalence of Long-COVID Syndrome is unknown; however, it may occur in up to one-in-three survivors or more depending on the depth of investigation and fidelity of diagnostic tools. A dedicated effort within the scientific, medical and lay communities to understand the natural history of
COVID-19 and its sequelae must be undertaken. Optimal patient care depends on it (Table 4).

Everything around us is filled with mystery and magic. I find this no cause for despair, no reason to turn for solace to esoteric formulae. On the contrary, our inability to find easy answers fills me with a fierce pride in our ambivalent biology ... with a constant sense of 
wonder and delight that we should be part of anything so profound.

Lyall Watson

\section{References}

1. Becker RC (2020) Anticipating the long-term cardiovascular effects of COVID-19. J Thromb Thrombolysis 50:512-524

2. Pietsch H, Escher F, Aleshcheva G, Baumeier C, Morawietz L, Elsaesser A, Schultheiss HP (2020) Proof of SARS-CoV-2 genomes in endomyocardial biopsy with latency after acute infection. Int J Infect Dis IJID Off Publ Int Soc Infect Dis 102:70-72

3. Donnelly JP, Wang XQ, Iwashyna TJ, Prescott HC (2021) Readmission and death after initial hospital discharge among patients with COVID-19 in a large multihospital system. JAMA 325:304-306

4. Dennis A, Wamil M, Kapur S, Alberts J, Badley AD, Decker GA, Rizza SA, Banerjee R, Banerjee A (2020) Multi-organ impairment in low-risk individuals with long COVID. medRxiv. https://doi. org/10.1101/2020.10.14.20212555

5. Datta SD, Talwar A, Lee JT (2020) A Proposed framework and timeline of the spectrum of disease due to SARS-CoV-2 infection: illness beyond acute infection and public health implications. JAMA 324:2251-2252

6. Kim JH, Levine BD, Phelan D, Emery MS, Martinez MW, Chung EH, Thompson PD, Baggish AL (2020) Coronavirus disease 2019 and the athletic heart: emerging perspectives on pathology, risks, and return to play. JAMA Cardiol. https://doi.org/10.1001/jamac ardio. 2020.5890

7. Greene DN, Wu AHB, Jaffe AS (2020) Return-to-play guidelines for athletes after COVID-19 infection. JAMA Cardiol 70:1902-1918

8. Phelan D, Kim JH, Elliott MD, Wasfy MM, Cremer P, Johri AM, Emery MS, Sengupta PP, Sharma S, Martinez MW, La Gerche A (2020) Screening of potential cardiac involvement in competitive athletes recovering from COVID-19: an expert consensus statement. JACC Cardiovasc Imaging 13:2635-2652

9. Wood E, Hall KH, Tate W (2020) Role of mitochondria, oxidative stress and the response to antioxidants in myalgic encephalomyelitis/chronic fatigue syndrome: a possible approach to SARS-CoV-2 'long-haulers'? Chronic Dis Transl Med. https://doi.org/10.1016/j. cdtm.2020.11.002

10. Stussman B, Williams A, Snow J, Gavin A, Scott R, Nath A, Walitt B (2020) Characterization of post-exertional malaise in patients with myalgic encephalomyelitis/chronic fatigue syndrome. Front Neurol 11:1025

11. Segal E, Zhang F, Lin X, King G, Shalem O, Shilo S, Allen WE, Alquaddoomi F, Altae-Tran H, Anders S, Balicer R, Bauman T, Bonilla X, Booman G, Chan AT, Cohen O, Coletti S, Davidson N, Dor Y, Drew DA, Elemento O, Evans G, Ewels P, Gale J, Gavrieli A, Geiger B, Grad YH, Greene CS, Hajirasouliha I, Jerala R, Kahles A, Kallioniemi O, Keshet A, Kocarev L, Landua G, Meir T, Muller A, Nguyen LH, Oresic M, Ovchinnikova S, Peterson H, Prodanova J, Rajagopal J, Rätsch G, Rossman H, Rung J, Sboner A, Sigaras A, Spector T, Steinherz R, Stevens I, Vilo J, Wilmes P (2020) Building an international consortium for tracking coronavirus health status. Nat Med 26:1161-1165

12. Smolinski MS, Crawley AW, Baltrusaitis K, Chunara R, Olsen JM, Wójcik O, Santillana M, Nguyen A, Brownstein JS (2015) Flu near you: crowdsourced symptom reporting spanning 2 influenza seasons. Am J Public Health 105:2124-2130

Publisher's Note Springer Nature remains neutral with regard to jurisdictional claims in published maps and institutional affiliations. 